\title{
Application of analytics in medical treatment cost optimization
}

\section{Introduction}

Medical treatment cost has increased continuously over a period of time. To afford basic medical treatment has become difficult for the general population. To minimize the basic medical treatment cost we need to improve the healthcare decision making capabilities. Different costs are associated with the medical treatment like inventory cost, supply chain cost, facility cost and service cost. Analytics techniques help to optimize all these costs. ${ }^{1}$ Optimization of all these costs help to minimize the medical treatment cost to the poor of poorest in the country. Healthcare decision making can be broadly classified into three categories namely; strategic, tactical and operational. ${ }^{2}$ Analytics techniques improve all the levels of decision making processes. Proposed framework can optimize the medical treatment cost that will help to improve the healthcare system of the developing countries.

\section{Framework}

\section{Analytics techniques used in healthcare decision making}

Descriptive analytics is the initial stage of data processing that leads to a summary of the available data to yield useful information (Figure 1). It also prepares the data for further analysis and provides information about the events that occurred. Predictive analytics is used to make predictions about the unknown future events. It uses the techniques of data mining and statistics to analyze current data and make predictions about future events. Prescriptive analytics is the final phase of application of analytics, which includes descriptive and predictive analytics. It anticipates what will happen, when it will happen and why it will happen. Further, it suggests decision options on how to take advantage of a future opportunity or mitigate a future risk and shows the implication of each decision option. ${ }^{3}$

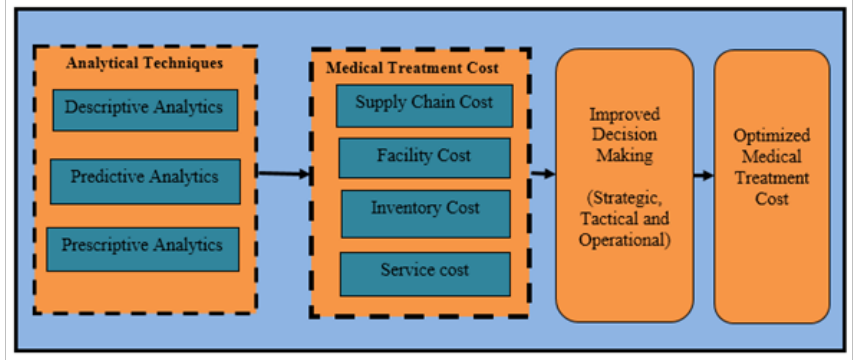

Figure I Framework of Medical Treatment Cost Optimization using Analytics.

\section{Medical treatment cost}

The cost of medical treatment to the end user in healthcare setup depends on the factors mentioned in fig 1 which is influenced by each other. Any change in the supply chain cost influences the facility and inventory cost and ultimately the service cost changes and gives a burden to the patient. ${ }^{4}$

\section{Decision making in healthcare}

Plays a vital role in optimizing cost of medical treatment. Strategic Decision Making (Time frame: Years) is an ongoing process that includes strategies to achieve goals based on observed
Volume 2 Issue 2 - 2018

Mohd Zuber
Department of Public Health, Saudi Electronic University,
Kingdom of Saudi Arabia

Correspondence: Mohd Zuber, Department of Public Health, College of Health Sciences, Saudi Electronic University, Kingdom of Saudi Arabia, Tel +966580223376, Email zubermohd786@gmail.com

Received: February 19,2018 | Published: April 19, 2018

outcomes. It involves defining long-term goals, relate to market forces and accomplish the firm's mission. When making strategic decisions, managers consider the impact that potential actions may have on the company, its competition and its market. This is used in healthcare planning and policy making, infrastructure development, establishment of new hospitals and medical colleges. ${ }^{1}$ Tactical Decision Making (Time frame: Months) are medium term, less complex decisions made by mid level managers. They follow on the strategic decisions and move ahead to meet the objectives stated in any strategic decision. ${ }^{1}$ Operational Decision Making (Time frame: Days) is facilitated by real time information. It is coordinated by dayto-day planning and can reduce overall operational cost and results in an integrated operational activities. It ensures that operational decision making is critical to the healthcare industry and worthy of executive and management focus. ${ }^{5}$

\section{Discussion}

The use of analytic techniques can help healthcare managers to monitor ongoing processes in the industry and prepare for the future challenges. The ongoing cost can be controlled at different levels and one can be prepared for the handling of upcoming adverse events. By the application of these techniques common people can avail an affordable treatment at the hospitals. Thus with the improvement of healthcare decision making capabilities the availability of providing cost effective treatment can be a reality. ${ }^{2}$

\section{Acknowledgement}

None.

\section{Conflict of interest}

The author declares there is no any conflict interest.

\section{References}

1. What Is Strategic Decision-Making? 2018.

2. Chapter 9: Marketing Information Systems.

3. Pedro M Kono, Barry Barnes. The Role of Finance in the StrategicPlanning and Decision-Making Process. A Peer Reviewed Journal Advancing Business Practice. 2010;13(1).

4. The Decision-Making Process.

5. Supporting Patients' Decision-Making Abilities and Preferences. 